\title{
The stress state in the three-layer open conical shell during of stability loss
}

\author{
Edward Tertel*, Piotr Kurylo, Wladyslaw Papacz
}

University of Zielona Góra, Faculty of Mechanical Engineering, Prof. Z. Szafrana 4, 65-516 Zielona Góra

\section{BIOGRAPHICAL NOTES}

Edward Tertel, Ing. PhD. was born in 1970 in Zagan, Poland. Since 2003 he works on the Faculty of Mechanical Engineering, University of Zielona Góra as an assistant professor. In 2003 he gained PhD in the field of mechanics at the Faculty of Mechanical Engineering and Management, Poznan University of Technology. He is an author or coauthor of publications in domestic and foreign journals and conference proceedings. His research work is focused on analyses of elastic-plastic stability and optimization of sandwich structures. Member of Polish Society of Theoretical and Applied Mechanics and Polish Society of Production Management.

Władysław Papacz, Ing. PhD., (1961) Since 2001 he has been working at the Faculty of Mechanical Engineering, University of Zielona Góra as an assistant professor. He graduated from the faculty of construction and operation of machines. His scientific and research work focused in the field of optimization and development of polymer composites structures. Member of Central European Composite Claster and Polish Scientific Society of Motorization.

Piotr Kurylo, PhD eng. was born in 1966 in Zielona Gora, Lecturer at University of Zielona Gora. Assistant Professor in Faculty of Mechanical Engineering University of Zielona Gora. Completed his doctorate at the University of Poznan. Member of the Faculty of Mechanical Engineering. Specialist professional engineering, engineering of the surface layer, automation and robotics, manufacturing technologies.

\section{KEY WORDS}

Stress, buckling, sandwich shells, stability, plasticity.

\section{ABSTRACT}

The paper presents an analysis of stress state in a sandwich open conical shells during of stability loss. The shells under consideration consist of lightweight core layer and two face-layers which are load-carrying. The thickness of those faces is assumed to be equal, and the thickness of the core is about $80 \%$ of the whole shell thickness. The load carrying faces are made of isotropic, compressible, work-hardening materials. The core layer is assumed to be elastic, incompressible in the normal z direction and it resists transverse shear only. The shell under consideration is loaded by lateral pressure and longitudinal force. Deformation of the shells within the plastic range is possible before buckling. To determine the stress state which occurs during stability loss, the stability equations have to be derived. With respect to the presented research, constitutive relations of the Nadai-Hencky deformation theory, alongside the HMH (Huber-MisesHencky) yield condition constitutive relations, are accepted in the analysis. 


\section{Introduction}

Thin-walled shells consisting of three layers have high structural efficiency and they are very interesting for a designers and builders of the lightweight, modern constructions. Those structures are more often used to design various structures (e.g. aerospace, buildings, tanks) and therefore these are well recognized in the scientific literature [1-4]. The main advantage of such structures is the load-toweight ratio which is comparatively high. In effect of that, the shells with high bending stiffness and high compressive buckling strength witch comparatively low weight can be obtained. The conical shell elements are often used in some kind of technical structures (e.g. plane and aerospace fuselage, train bodies). Therefore, in particular applications, a sandwich conical shell element under combined external loads may provide for interesting case studies. Stability of cylindrical and conical shells is considered by many authors. Research works refers to single-layer shells [5-7], as well as to the bi-layered [8,9] and sandwich shells [10-13]. Various methods of sandwich structures analysis are presented in $[3,4]$ and a large reference list is given there. Some empirical results concerning conical shells subjected to external uniform pressure are presented in [7]. In [1,2] an analysis of conical shells under various loading is presented. Interesting, new method of empirical measurement of strains and stress fields in the loaded structures is presented in Ref. [14]. In the majority of publications some initial assumptions about changes in stress values are accepted. Most of publications assume the active loading process (Shanley approach) [10-13].

Article shows the possibility of determining the stress changes during of stability loss. This allows for the adoption an appropriate method of loads of the construction (constant load or active load approach). An analysis of the stress changes of sandwich conical shells under combined loading is the main objective of this paper.

\section{Basic assumptions}

Several assumptions must be accepted at first. The analyzed object is a sandwich conical shell element. The shell consists of two thin face-layers and one core layer (Figure 1). In this consideration the face-layers are of equal thicknesses and are made of the same material which is compressible and isotropic. The other considerations especially for unsymmetrical shells could be seen in the previous publications $[11,12]$. The core layer is made of lightweight material and is assumed to be elastic, incompressible in the normal $z$ direction and it resists transverse shear only. The symmetry surface of the shell is taken as the reference surface.

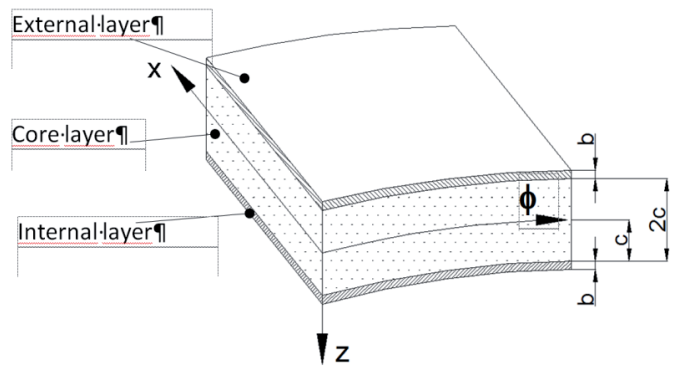

Fig. 1: The layered shell with the coordinate system.

The several additional assumptions, which have been taken with respect to the foregoing model, are as follows:

- the shell is shallow and thin-layered,

- the general theory of thin-layered shells and geometrically nonlinear theory and elastic-plastic properties of the faces are obligatory,

- the Kirchhoff-Love hypothesis is valid within the entire cross-section of the shell and the displacement in normal $z$ direction does not depend on the $z$ coordinate,

- the prebuckling stress state is the membrane one, - the post-buckling stress state could be elastic, elastic-plastic or plastic,

- the bilinear stress-strain relation for the face-layers is accepted and the constitutive relations in the analysis are those of the Nadai-Hencky deformation theory with the HMH yield condition,

- there is no imperfections in the considered shell.

\section{Loading and stresses}

There are two different types of loads acting on the shell under consideration. The first one is the longitudinal forces $N_{a}, N_{b}$ applied at the edges of the shell and the second one is the surface pressure $q$ directed perpendicularly to the shell main surface (Figure 2).

According to the main assumptions, there is a membrane stress state in the shell. Internal forces in that stress state vary on $\mathrm{x}$-coordinate and are described as follows:

\section{- from the compressive axial forces:}


$N_{x N}=\sigma_{x N} \cdot 2 b=-N_{a} \frac{x_{1}}{x}$,

$N_{\phi N}=0$,

$T_{x \phi N}=T_{\phi x N}=0$,

\section{from the lateral pressure:}

$N_{x p}=\sigma_{x p} \cdot 2 b=\frac{1}{2} q \cdot x \cdot \operatorname{tg}(\alpha) \cdot\left[\left(\frac{x_{1}}{x}\right)^{2}-1\right]$,

$N_{\phi p}=\sigma_{\varphi p} \cdot 2 b=-q \cdot x \cdot \operatorname{tg}(\alpha)$,

$T_{x \phi}=T_{\phi x}=0$.

\section{- from the total two-parametrical load:}

$$
\begin{aligned}
& N_{x}=N_{x N}+N_{x p}=\sigma_{x} \cdot 2 b= \\
& =\frac{1}{2} q \cdot x \cdot \operatorname{tg}(\alpha) \cdot\left[\left(\frac{x_{1}}{x}\right)^{2}-1\right]-N_{a} \frac{x_{1}}{x}, \\
& N_{\varphi}=N_{\varphi N}+N_{\varphi p}=\sigma_{\varphi} \cdot 2 b=-q \cdot x \cdot \operatorname{tg}(\alpha), \\
& T_{x \varphi}=T_{\varphi x}=0 .
\end{aligned}
$$

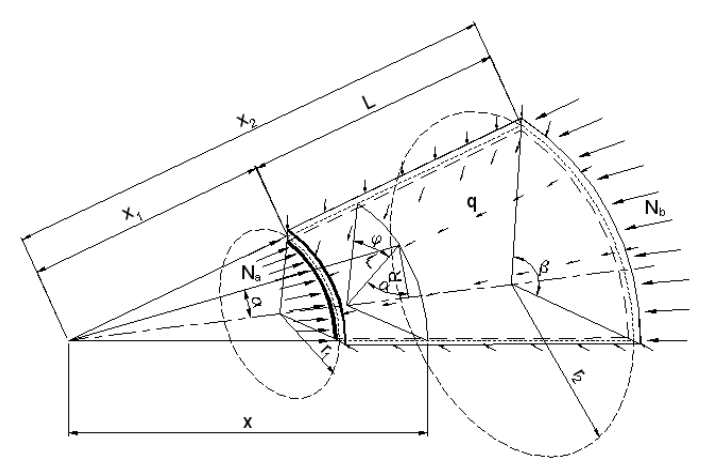

Fig. 2: The model of the shell under consideration, $q, \mathrm{Na}, \mathrm{Nb}-\mathrm{ex}$ ternal loads.

The limitation of the Nadai-Hencky deformation theory which is used in this work is the condition of proportional loading. Because of this the parameter $\kappa$, which represents the ratio between the lateral and longitudinal load is introduced:

$\kappa=\frac{N_{a}}{q \cdot x_{1}}=$ const.

The stresses in the pre-buckling state of stress can be accordingly expressed by the external forces:

$$
\sigma_{x}=\frac{-q \cdot x \cdot \operatorname{tg}(\alpha)}{4 b} \cdot k,
$$

$\sigma_{\varphi}=\frac{-q \cdot x \cdot \operatorname{tg}(\alpha)}{2 b}$,

$\tau_{x \phi}=0$,

and the effective stress:

$\sigma_{i}=\frac{q \cdot x \cdot \operatorname{tg}(\alpha)}{4 b} \sqrt{k^{2}-2 k+4}$,

where $k=\left(1-\left(\frac{x_{1}}{x}\right)^{2} \cdot\left(1-\kappa \frac{2}{\operatorname{tg}(\alpha)}\right)\right)$.

\section{Stability equations and equilibrium paths}

To calculate the effective stress during the stability loss, it is necessary to calculate the critical values of the external loads. This goal was achieved and described in previous works $[11,12]$. In those papers the stability equations of sandwich conical shells under combined load were analyzed and the procedure for obtaining the equilibrium paths is described in detail. Therefore in this work the calculation procedure will be described only in a general manner. To derive the stability equations a variation, strain energy method was accepted and the Ritz method was used to solve the stability equations.

The geometrical relations between the displacement components: $u, v, w$ of the arbitrary point of the shell and the displacements of the points on the middle surfaces of the specific faces $u_{1}, u_{2}$, $v_{1}, v_{2}$ (Figure 3) were described by following the so called broken line approach.

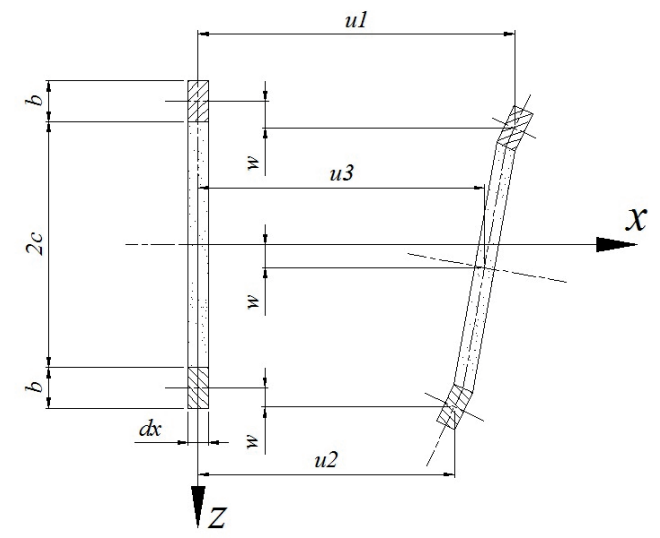

Fig. 3: The displacements of the sandwich shell element.

The displacements for the arbitrary points of the shell could be described separately for: 


\section{- the upper face-layer $[-c-b \leq z \leq-c]$ :}

$$
\begin{aligned}
& u=u_{\alpha}+u_{\beta}-\left(z+c+\frac{b}{2}\right) \frac{\partial w}{\partial x}, \\
& v=v_{\alpha}+v_{\beta}-\left(z+c+\frac{b}{2}\right) \frac{1}{r} \frac{\partial w}{\partial \varphi},
\end{aligned}
$$

\section{- the core-layer $[-\mathrm{c} \leq \mathrm{z} \leq \mathrm{c}]$ :}

$$
\begin{aligned}
& u=u_{\alpha}-\frac{z}{c}\left[u_{\beta}-\frac{b}{2} \frac{\partial w}{\partial x}\right], \\
& v=v_{\alpha}-\frac{z}{c}\left[v_{\beta}-\frac{b}{2 r} \frac{\partial w}{\partial \varphi}\right] .
\end{aligned}
$$

\section{- the lower face-layer $[\mathrm{c} \leq \mathrm{z} \leq \mathrm{c}+\mathrm{b}]$ :}

$$
\begin{aligned}
& u=u_{\alpha}-u_{\beta}-\left(z-c-\frac{b}{2}\right) \frac{\partial w}{\partial x}, \\
& v=v_{\alpha}-v_{\beta}-\left(z-c-\frac{b}{2}\right) \frac{1}{r} \frac{\partial w}{\partial \varphi},
\end{aligned}
$$

Where: $u_{\alpha}, u_{\beta}, v_{\alpha}, v_{\beta}$ are relations between displacements of the points on the middle surfaces of the specific faces $[1,2]$ :

$$
\begin{array}{ll}
u_{\alpha}=\frac{u_{1}+u_{2}}{2}, & u_{\beta}=\frac{u_{1}-u_{2}}{2}, \\
v_{\alpha}=\frac{v_{1}+v_{2}}{2}, & v_{\beta}=\frac{v_{1}-v_{2}}{2}
\end{array}
$$

The strains and changes in curvature are expressed depending on the displacements as described above, using non-linear geometrical relations given in [1].

The effective stress in the shell faces can exceed the yield stresses for the material of these faces. When this occurs the constitutive relations of the Nadai-Hencky deformation theory were used to obtain stability equations. The basic form of the equations of this plasticity theory is as follows:

$$
\varepsilon_{i j}=m\left(\varepsilon_{i}\right) \sigma_{i j}+\left[\frac{1}{3 K}-m\left(\varepsilon_{i}\right)\right] \sigma_{m} \delta_{i j}
$$

Where: $\sigma_{\mathrm{m}}$ is average stress, $\delta_{\mathrm{ij}}$ is the Kronecker delta, $K, \mathrm{~m}\left(\varepsilon_{\mathrm{i}}\right)$ are the material constants.

The internal forces and moments developed by buckling were derived and can be described in a matrix form:

$$
\begin{aligned}
& {\left[\begin{array}{l}
\delta N_{x(j)} \\
\delta N_{\varphi(j)} \\
\delta T_{(j)}
\end{array}\right]=\left[\begin{array}{rrr}
B_{11(j)} & B_{12(j)} & -B_{13(j)} \\
B_{21(j)} & B_{22(j)} & -B_{23(j)} \\
-B_{31(j)} & -B_{32(j)} & B_{33(j)}
\end{array}\right] \times\left[\begin{array}{l}
\delta \varepsilon_{x(j)} \\
\delta \varepsilon_{\varphi(j)} \\
\delta \gamma_{x \varphi(j)}
\end{array}\right]} \\
& {\left[\begin{array}{l}
\delta M_{x(j)} \\
\delta M_{\varphi(j)} \\
\delta H_{(j)}
\end{array}\right]=\left[\begin{array}{rrr}
-D_{11(j)} & -D_{22(j)} & D_{23(j)} \\
-D_{21(j)} & -D_{22(j)} & D_{23(j)} \\
D_{31(j)} & D_{32(j)} & -D_{33(j)}
\end{array}\right] \times\left[\begin{array}{l}
\delta \kappa_{x(j)} \\
\delta \kappa_{\varphi(j)} \\
\delta \kappa_{x \varphi(j)}
\end{array}\right]}
\end{aligned}
$$

Where: $j=1$ for the upper face-layer, $j=2$ for the lower face-layer.

The coefficients in Eqs.(12) are the local stiffnesses that follow the Nadai-Hencky deformation theory of plasticity.

A set of the stability equations, expressed by the displacements does not have an explicite solution. The virtual work principle and the strain energy methods comprise a basis for obtaining equilibrium equations for the considered shell. As far as a sandwich shell, the total strain energy is the sum of the strain energy of the specified layers. If the potential of external loads is represented by $L_{z}$ we have the following relations:

$$
\delta U_{p}=\delta\left(U_{w}^{(1)}+U_{w}^{(2)}+U_{w}^{(3)}+L_{z}\right)=0 .
$$

Where: the particular superscripts are related to the shell layers: ${ }^{(1),(2)}$ - the face layers, ${ }^{(3)}$ - the core layer.

The terms in Eq.(13) related with the strain energy were described in $[11,12]$.

The considered shell is free supported. The displacements functions must satisfy the boundary conditions concerning the shell under consideration: deflections at the edges of the shell are equal to zero, there are no displacements along the supports and there are no relative displacements of the both layers of the shell at the edges. Bearing in mind the above conditions the following displacements functions were accepted [1, 11-13]:

$$
\begin{aligned}
& u_{\alpha}(x, \varphi)=A_{2} r^{2} \cos \left(k\left(x-x_{1}\right)\right) \sin (p \varphi), \\
& u_{\beta}(x, \varphi)=A_{3} r^{2} \cos \left(k\left(x-x_{1}\right)\right) \sin (p \varphi), \\
& v_{\alpha}(x, \varphi)=A_{4} r^{2} \sin \left(k\left(x-x_{1}\right)\right) \cos (p \varphi),(14) \\
& v_{\beta}(x, \varphi)=A_{5} r^{2} \sin \left(k\left(x-x_{1}\right)\right) \cos (p \varphi), \\
& w(x, \varphi)=A_{1} r^{2} \sin \left(k\left(x-x_{1}\right)\right) \sin (p \varphi) .
\end{aligned}
$$

Where: $A_{i}$ are free parameters to be determined, $k$, $p$ are constants. 
Using the above displacement relations in the nonlinear geometrical relations, the full description of the shell potential energy $U_{p}$ is obtained. Next the Ritz method was applied. This method requires that the partial derivatives of the total potential energy of the shell with respect to parameters $A_{i}$ are zero. Finally, after some transformations a set of algebraic equations for the considered shell were obtained. The general matrix form of these equations is as follows:

$$
\left[\begin{array}{ccccc}
a_{11}+a_{11 p} & a_{12} & a_{13} & a_{14} & a_{15} \\
a_{21} & a_{22} & a_{24} & a_{24} & a_{25} \\
a_{31} & a_{32} & a_{33} & a_{34} & a_{35} \\
a_{41} & a_{42} & a_{43} & a_{44} & a_{45} \\
a_{51} & a_{52} & a_{53} & a_{54} & a_{55}
\end{array}\right] \times\left[\begin{array}{c}
A_{1} \\
A_{2} \\
A_{3} \\
A_{4} \\
A_{5}
\end{array}\right]=\left[\begin{array}{c}
b_{11} A_{1}^{2}+b_{12} A_{1}^{3}+b_{13} A_{1} A_{2}+b_{14} A_{1} A_{3}+b_{15} A_{1} A_{4}+b_{16} A_{1} A_{5}+b_{17} \\
b_{21} A_{1}^{2} \\
b_{31} A_{1}^{2} \\
b_{41} A_{1}^{2} \\
b_{51} A_{1}^{2}
\end{array}\right]
$$

Where: $a_{i j}, b_{i j}$ are coefficients of the set of equations which depend on geometrical parameters of the shell, physical properties, buckling form, and external loading.

The set of equations Eqs. (15) solved with respect to the free parameter $A_{l}$ of the deflection function Eqs. (14), and following some transformations and simplifications, provides the final solution in the form of a non-linear algebraic equation:

$$
q=\frac{e_{1} A_{1}+e_{2} A_{1}^{2}+e_{3} A_{1}^{3}}{A_{1} e_{4} \kappa+e_{5}}
$$

Where: $e_{i}$ are coefficients of the stability equation which are very complicated in form and depend on the geometrical parameters, physical properties, buckling form and external loads acting upon the shell, $\kappa$-ratio of proportional loading.

It is not possible to find an explicite solution to Eq. (16) because the coefficients in this equation depend on the external loads and should be considered as a variable. A solution could be obtained numerically, using iterative methods. Thus, the iterative methods have been applied and a special computer algorithm for the numerical calculations and elastic-plastic analysis has been developed. In Figure 4. could be seen the sample diagrams of the equilibrium paths made for different value of the shell inclination angle $(\alpha)$.

\section{The stress state during of stability loss}

The objective of this work is to determine the stress state in the face layers of the shell under consideration (Figure 1) during the stability loss. Based on the known values of the critical loads we can calculate the stresses occurring in the face layers of the shell. The calculations are performed

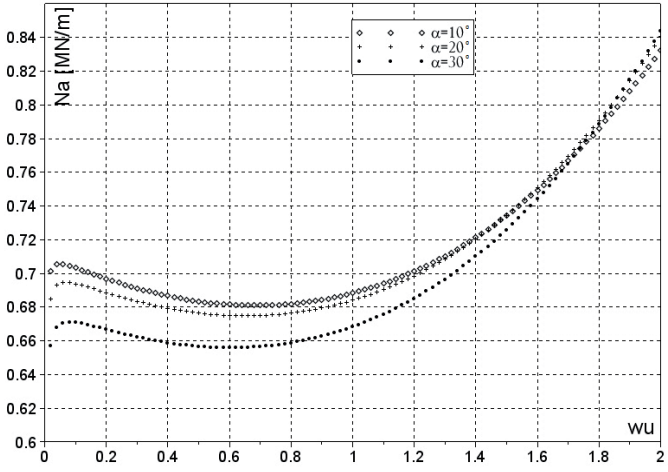

Fig. 4: Equilibrium paths for various value of the shell inclination angle.

for increasing values of the parameter wu which describes the shell deflection. It is possible to describe the variability of the stress along the $x$-coordinate.

In the performed calculations, the following input data were accepted.

\section{The physical parameters:}

- the load carrying layers:

- Young's modulus (E-210000 MPa)

- Tangent modulus (Et-30000 MPa)

- Poisson's ratio $(\nu-0.3)$

- Yield stress $\left(\sigma_{p}-240 \mathrm{MPa}\right)$

- the core layer:

- Shear modulus (G3-26 MPa)

\section{The geometrical parameters:}

- Length of the shell $(L-0.5 \mathrm{~m})$

- Average radius of the shell $\left(R_{s}-2 m\right)$

- Thickness of core layer $(2 c-10 \mathrm{~mm})$

- Thickness of the face layers $(b-1 \mathrm{~mm})$

- Inclination angle $\left(\alpha-30^{\circ}\right)$

- Radial angle $\left(\beta-30^{\circ}\right)$

The overall parameters: 
- Load ratio ( $\kappa-500)$

- Number of half-waves along the shell length $(m-1)$

- Number of circumferential half-waves ( $n$ - 1$)$

- Maximum of the shell deflection parameter $(W u(\max )-2)$

The shell deflection parameter is defined as follows: $\quad w_{u}=w /(2 b+2 c)$.

The surface on Figure 5 represents the changes in the stress depending on the $x$-coordinate measured along the shell and for various values of the shell deflection. The relation: effective stress (sig) - deflection parameter $\left(w_{u}\right)$ - $\mathrm{x}$-coordinate on the shell $\left(L_{x}\right)$ is the base to present the obtained results and discuss them. For better visualization Figures $6 \mathrm{a}$ and $6 \mathrm{~b}$ shows separately a family of curves $\operatorname{sig}\left(w_{u}\right)$ and $\operatorname{sig}\left(L_{x}\right)$ respectively. As may be noted the shape of the curves in Figure 6a resembles the shape of the equilibrium paths shown in Figure 5. The effective stress initially increases intensively. When the deflection parameter reaches a certain value ( $w_{u}=0,08$ for analyzed shell) the effective stress reaches the extreme point (maximum) and the process of stability loss is initiated. Then can be observed drop of the effective stress. Then after reaching the next extremum (minimum), the effective stress increases again. It is noteworthy that the position of the extremal points is not dependent on the $x$-coordinate. On the other hand the effective stress value depends on the $x$-coordinate what can be clearly seen in Figure $6 \mathrm{~b}$. If the $\mathrm{x}$-coordinate increases (towards to the base of the cone) the effective stress drops linearly. The relative change of the effective stress remains constant and for the considered shell reaches a value of $11.8 \%$.

(a)

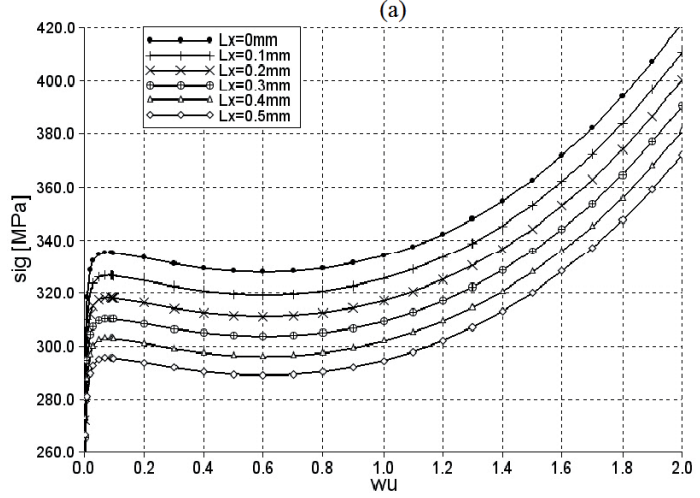

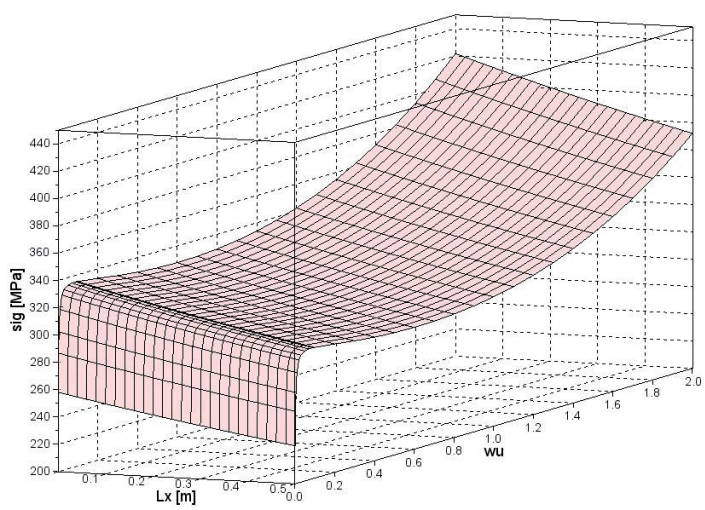

Fig. 5: The effective stress versus the deflection and the $x$ coordinate: sig - the effective stress; wu - the shell deflection parameter; $L_{x}$ - the length of the shell measured along $x$-coordinate $\left(L x=x-x_{1}-\right.$ see Figure 1$)$

\section{Conclusions}

The objective of this paper is to analyze of the state of stress in the three-layer conical shell during the stability loss. For the shell with the parameters accepted in the analysis, the stability loss process occurs in the elastic-plastic or plastic state of stress. The work presents the changes in the effective stress during the stability loss on the entire length of the shell. Presented results show that during the stability loss, the effective stress may decrease over the whole length of the shell (unloading process). If this occurs, it seems to be more appropriate to use the so called constant load concept (EngesserKarman approach) which takes into consideration the material unloading and hardening after the stability loss. The constant load approach is a better approach from theoretical point of view because it takes into consideration the material unloading.

(b)

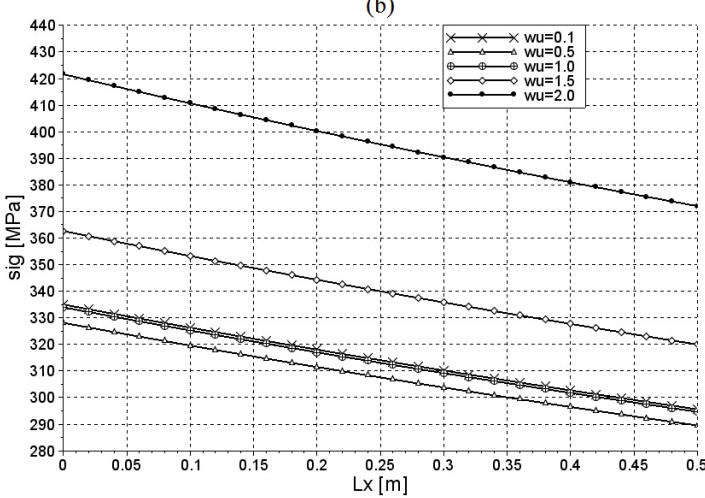

Fig. 6: (a) The effective stress versus the deflection for selected values of the x-coordinate. (b) The effective stress versus the x-coordinate for selected values of the deflection. 
This approach will be considered in subsequent studies.

\section{References}

[1] Zielnica J (2001). Stability of elastic-plastic shells (in Polish). Poznan Uni. of Tech.

[2] Magnucki K, Ostwald M (2001). Stability and optimization of sandwich structures, (in Polish). -Red. of collective work, Poznan Uni. of Tech - Uni. of Zielona Gora.

[3] Hohe J, Librescu L (2004). Advances in the structural modeling of elastic sandwich panels. Mechanics of Adwanced Materials and Structures, 11:4-5, pp. 395-424.

[4] Vinson J R (2001). Sandwich Structures. Applied Mechanics Reviews, vol. 54, no. 3, pp. 201-214.

[5] Błachut J (2011). On elastic-plastic buckling of cones. Thin Walled Structures, no .49, pp.45-52.

[6] Błachut J, Ifayefunmi O (2010). Plastic buckling of conical shells. Journal of Offshore Mechanics and Arctic Engineering, 132(4), 041401-1 - 041401-11.

[7] Golzan B S, Showkati H (2008). Buckling of thin-walled conical shells under uniform external pressure. Thin-Walled Structures, no. 46, pp. 516-529.

[8] Paczos P, Zielnica J (2007). Critical load of a bilayered orthotropic elastic-plastic conical shell with the change of the shell basic surface location. Thin-Walled Structures, no. 45, pp. 911-915.

[9] Paczos P, Zielnica J (2008). Stability of orthotropic elasticplastic open conical shells. Thin-Walled Structures, no. 46, pp. 530-540.

[10] Jaskula L, Zielnica J (2011). Large displacement stability analysis of elastic-plastic unsymmetrical sandwich cylindrical shells. Thin Walled Structures, 49, 5, pp.611-617.

[11] Tertel E (2013). Stability of elastic-plastic sandwich conical shells with a change in the thickness of face-layers. Journal of vibroengineering, Vol. 15, pp. 684-692.

[12] Tertel E, Cyganiuk J, Kuryło P (2011). Buckling of elastic-plastic sandwich conical shells - theoretical studies. International Journal of Applied Mechanics and Engineering, Vol. 16, No. 1, pp.129-140.

[13] Zielnica J (2003). Non-linear stability of elastic-plastic conical shell under combined load. Journal of Theoretical and Applied Mechanics, no. 41(3), pp. 693-709.

[14] Trebuna F, Frankovsky P, Bocko J, Pastor M (2011) New posibilities of using PhotoStress method. Acta Mechanica Slovaca, 15(4), pp. 44-51.

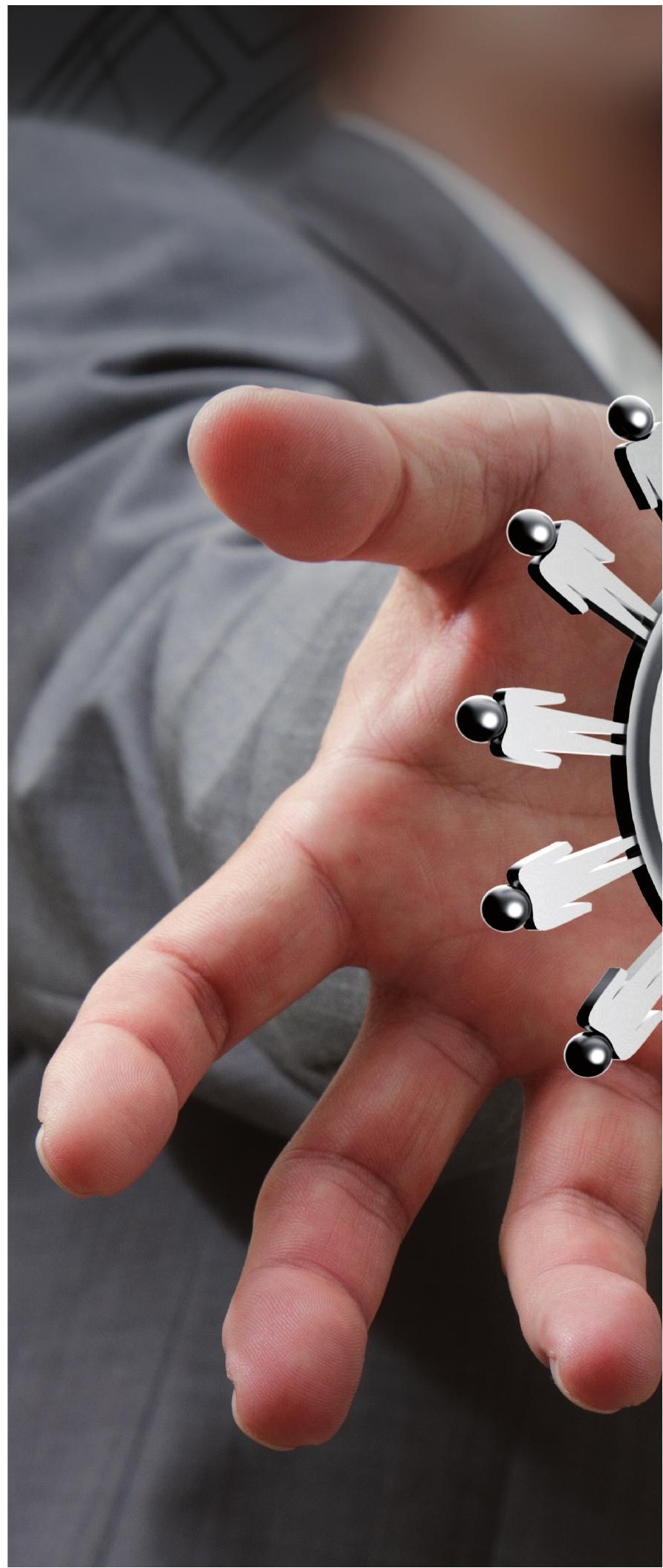


